\title{
Application of PDCA Management Theory in Railway Maintenance
}

\author{
Mao Jianhong \\ School of Civil and Architectural Engineering, East China Jiaotong University, Nanchang, Jiangxi, \\ China
}

11223@163.com

Keywords: PDCA; Railway Maintenance; Total Quality Management

\begin{abstract}
Implementation of railway maintenance job could not only greatly improve the service life of the railway, but is of great significance to the safety of railway transportation. In the article, combined with the basic connotation of PDCA cycle management, we propose some strategies to improve the efficiency of railway maintenance.
\end{abstract}

\section{Introduction}

Railway has become an important infrastructure of a country, and railway maintenance is an important work for the railway to play its lifeline role. However, for a long time, due to long waste of time and huge cost, railway maintenance becomes a major expenditure on railway operations and the most important part of the railway management department.

All the time, many experts and scholars have been looking for ways to improve the work efficiency and working methods for railway maintenance, but the improvement effect is always reflected in machinery replacement and adopting modern equipment, instead of improvement of working methods.

Railway maintenance is complicated system engineering, including signal, power supply, communication and other operations, and it requires the coordination of many workers. Due to its high requirements in security, we have to pay more attention in quality supervision and control. Here we employ the PDCA cycle management method in railway maintenance work, and focus on how to formulate effective working mechanism, so as to promote communication and coordination between each maintenance teams.

\section{PDCA Cycle Management Method}

Connotation of PDCA. PDCA cycle is also called Deming cycle. PDCA cycle is a logical working procedure that can be effective in any activity, especially in quality management activities. The basic procedure of PDCA management method is as follow:

a) Plan: it includes the formulation of the guidelines, objectives and activity arrangements;

b) Do: it refers to specific implementation process, and achieves the planned content;

c) Check: it is to summarize the results of the implementation plan, so as to determine the implementation effect and find out the problems;

d) Action: according to the checked out situation, on the one hand, standardized the success experiences to draw up operating instructions; on the other hand, summed up the lessons of failure, so as to avoid it next time. As for the problems that have not been solved, could be passed on to the next PDCA cycle to solve.

The four stages of PDCA cycle is shown in Fig. 1: 


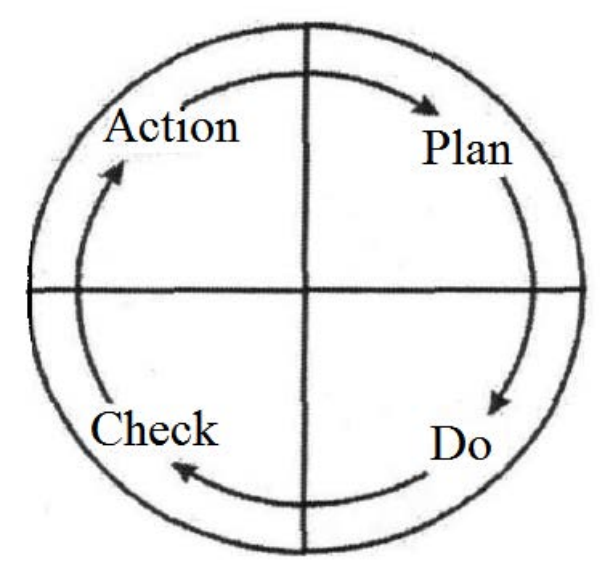

Fig. 1. Procedure of PDCA management method

\section{Characteristics of PDCA.}

(1) Back and forth

The four processes PDCA cycle is back and forth, instead of running just one time. When one cycle ends, some of the problems will be solved, while some other problems have not been solved, or new problems appear. Therefore, sometimes it needs one more cycle.

(2) Large ring with a small ring

Similar to the planetary gear train, the relationship between the overall operation system of an organization and its internal subsystem is organic logic assembly like large ring with a small ring.

(3) Stepped up

PDCA cycle is not stay at a level. In the process that problems are continually solved, the management level would be promoted step by step.

\section{PDCA Cycle Management Strategies in Railway Maintenance}

Plan. As an important part of railway maintenance management, plan formulation is the standard and guarantee for the smooth implementation of maintenance work. From distribution of personnel and machinery, to division of work responsibilities and determination of work standards, it needs to be fully considered from the start, so as to make a realistic and operational plan.

According to the characteristics of railway maintenance management, and based on the basic principle of PDCA cycle, we had better focus on the following aspects when make a plan:

(1) Give full consideration to the road characteristics and natural conditions

Due to the use of integrated work teams to segment the maintenance of the work, should try to reasonably allocate the maintenance section by a quantitative calculation, and then assign personnel and mechanical equipment in strict accordance with the workload and the nature of work, so as to realize optimal utilization of resources under the condition that the maintenance task is successfully completed. In addition, in the formulation of the annual work plan, the key and seasonal maintenance work should be arranged according to the characteristics of seasonal climate and the need of railway operation, to prevent the occurrence of line problems.

(2) Set reasonable skylight time

Skylight means the time that set aside for construction and maintenance operations. Currently, the skylight in some countries with higher railway management level is generally $2 \sim 6$ hours, however, due to the less railway line and the larger operation density in China, it is often difficult to guarantee a stable and adequate skylight time. But on the other hand, because of the mechanization degree of railway maintenance work in our country is not high, so it requires longer skylight. 
In response to this situation, at the same time that promote mechanization operation and reduce skylights time requirements, set reasonable skylight to ensure that maintenance and repair work smoothly, combined with the experience data and the change of train operation frequency and traffic volume, take into consideration of the impact of skylight time on train operation, and analyze the Working hours required by the integrated team, so that not only meet the needs of maintenance work, but also reduce its influence on the time and frequency of train operation.

(3) Draw lessons for advance prevention

PDCA cycle itself is a process of continuous improvement, while plan is the starting point for each level, and is also the key factor to determine the whole management level. The implementation of advance feedback require the accumulation of long and constant empirical data, with continuous practice and analysis, and analyze the root of the problem and find out the internal rules, so as to make a more workable plan to prevent the recurrence of the problem.

Do. Specific implementation process is a stage with the longest time, the maximum workload and the hardest management difficulty. For railway maintenance work, maintaining good equipment operation status is the basic and foundation to ensure long-term stability of railway transportation.

In the specific program implementation process, we should focus on the following aspects:

(1) Construct a high standard demonstration segment

In a particular line section, conduct planned, arranged and organized centralized maintenance, and construct high standard demonstration segment one by one, and eventually all equipment meet the standards across the board.

In the construction process of the demonstration segment, each integrated team should concentrate the resources and cooperate with each other, and provide effective technical support for the all-round development of three-dimensional conservation work.

(2) Implement condition-based maintenance (CBM)

Based on the deep familiarity with equipment performance, internal structure and operation principle, by the means of observing the appearance, measuring the dimensions and testing the equipment testing, to grasp the actual situation of the equipment and judge the hidden trouble, so that timely tackle the equipment failure.

In addition, constantly revised and update the original job content, standards, periodic maintenance mode. Particularly, in terms of maintenance job content, pay more attention to the status of the equipment, and avoid unnecessary maintenance, to maintain the overall quality of the equipment in a balanced and complete state.

(3) Strengthen inspection and assessment

Promote the guidance, supervision of various maintenance jobs, and meanwhile strengthen inspection and assessment mechanism. Include the work implementation situation into human resource assessment system, as the key basis to assess the work performance of the members of each comprehensive work team. The main objective lies in enhancing employees' enthusiasm for work, and cultivating the sense of competition among the teams.

Check. Insist on stereoscopic maintenance, and set up a leading group to take responsible for the formulation and implementation of stereoscopic maintenance work. Guide the quality and progress of the renovation of the professional team in a timely manner, to ensure the steady implementation of the stereoscopic maintenance work.

(1) Problems and deep reasons in the implementation process

a) Some employees don't have a full understanding on the concept of stereoscopic maintenance, and lack of comprehensive awareness and persistent urgency and responsibility sense, which causes resulted in slow progress in maintenance work. 
b) Currently, the standard for stereoscopic maintenance is not high, leading to repetitive operation or blind area in railway maintenance work; some working teams are not clear about the work ideas, without plan and institutional guarantee.

c) In stereoscopic maintenance, the labor productivity has not been put into staff quantitative assessment, so staff enthusiasm is not high and the stereoscopic maintenance effect is not significant.

(2) Internal three-level control

a) The first-level control is the internal control on each maintenance team. Through all kinds of rules and regulations and incentives, to carry out the specific requirements of the maintenance work, and guide every worker to fulfill their duties.

b) The second-level control is the control between professional teams. Build good communication mechanism among maintenance teams, and employ matrix structure to construct stereoscopic maintenance groups. Every group is responsible for the maintenance job of certain zone.

c) The third-level control refers to senior managers' control on the entire maintenance area under its jurisdiction. Through performance evaluation and quality inspection on every professional teams and groups, sort out the corresponding responsibilities and obligations.

For the control in railway maintenance implementation process, it can be divided into immediate feedback control and advance feedback control.

Action. In action stage, we need to integrate the jobs in the former three stages, and summarize the best way to solve the problem, and then develop standards and norms with high practical value.

(1) Establish comprehensive database and provide decision basis

With the increasing speed of railway and the improvement of railway traffic volume, the requirements on the measurement method and maintenance of track geometric state continues to increase. To conform to the trend of automated maintenance management, when China's railway departments developed maintenance plans and work order, should pay attention to the combination of computer technology for scientific analysis and prediction. Thus, it requires to collect a large amount of data, including geometry size, track types, train speed, track stress and so on, to build corresponding database, and then carry out data processing and analysis with computer.

(2) Improve the collection and processing of information.

Currently, in the railway maintenance work in our country, we often employ organization reporting system to achieve information communication. With such communication way, some valuable data and experience cannot be effectively preserved, and moreover, this will cause great loss of information in the information transmission process.

Therefore, combining the advanced experience of foreign countries, we could establish specialized information acquisition and processing sector, and set up specialized information working team. The team members are responsible for disaggregated data collation, and input to the database according to uniform standard format.

\section{References}

[1] Shan H E. Discussion on Quality Management of High-speed Railway Permanent Way Management and Inspection[J]. Railway Transport \& Economy, 2013.

[2] Zhang F. Discussion on the Quality Management of Railway Transport[J]. Railway Quality Control, 2008. 
[3] Liu W, Liang G Q, Hu J B. Continuous improvement for lean maintenance of equipment based on PDCA[J]. Modern Manufacturing Engineering, 2008.

[4] Wang L. Application of PDCA to the quality management of standard penetration test[J]. Hydrogeology \& Engineering Geology, 2012, 39(2):85-87.

[5] Ai-Min L I, Fang S Y, Min-Di W U. Application of PDCA Method in Quality Management for Equipment Maintenance[J]. Journal of Sichuan Ordnance, 2011. 